\title{
Investigation of Hammering Sound Frequency to Prevent Intraoperative Fracture during Total Hip Arthroplasty
}

\author{
Rina Sakai ${ }^{1,2}$ (D) Tatsuki Kitazato ${ }^{2}$, Katsufumi Uchiyama ${ }^{2,3}$, Kazuhiro Yoshida1,2, \\ Takeaki Yamamoto ${ }^{4}$, Naonobu Takahira' ${ }^{1,2}$, Masanobu Ujihira ${ }^{1,2}$
}

${ }^{1}$ Department of Medical Engineering and Technology, School of Allied Health Sciences, Kitasato University, Sagamihara City, Japan; ${ }^{2}$ Kitasato University Graduate School of Medical Sciences, Sagamihara City, Kanagawa, Japan; ${ }^{3}$ Department of Orthopedic Surgery, School of Medicine, Kitasato University, Sagamihara City, Japan;

${ }^{4}$ Department of Orthopedic Surgery, School of Medicine, St. Marianna University, Kawasaki City, Kanagawa, Japan

Correspondence to: Rina Sakai, rinax@kitasato-u.ac.jp

Keywords: Total Hip Arthroplasty, Periprosthetic Fracture, Cementless Stem, Hammering Sound, Peak Frequency Received: September 8, 2021

Accepted: October 26, 2021

Published: October 29, 2021

Copyright () 2021 by author(s) and Scientific Research Publishing Inc.

This work is licensed under the Creative Commons Attribution International License (CC BY 4.0).

http://creativecommons.org/licenses/by/4.0/

\section{(c) (i) Open Access}

\section{ABSTRACT}

Background: The 1st peak frequency of the hammering sound in total hip arthroplasty may serve as an evaluation index to prevent intraoperative fracture. Fixation of the stem and femur cannot be acquired unless the 1st peak frequency of hammering the stem into the femur stabilizes, and fixation can be judged as acquired when the 1st peak frequency becomes constant. To investigate whether the environmental sound in the operating room can be differentiated from the hammering sound of total hip arthroplasty, the 1st peak frequency of the hammering sound when impacting the stem into the femur with a hammer was identified. Method: The hammering sound of impacting the stem into a biomechanical test material through an impactor was analyzed using a fast Fourier transform analyzer. Environmental sound in the operating room was simulated and the 1st peak frequency of the sound on collision between the operator's voice and the surgical instrument was measured. The 1st peak frequency of hammering sound was compared between patients indicated for total hip arthroplasty and healthy individuals to investigate whether there is a difference due to bone quality. Results: The natural frequency of the impactor was $3.41 \pm 0.05 \mathrm{kHz}$, and the 1 st peak frequency of the femur, stem, and impactor was $2.43 \pm 1.45 \mathrm{kHz}$. The 1 st peak frequency of hammering sound on simulated femur in patients indicated for total hip arthroplasty was $2.98 \pm 0.73 \mathrm{kHz}$ and that in healthy individuals was $2.15 \pm 0.32 \mathrm{kHz}$. This suggested that the hammering sound in total hip arthroplasty-indicated patients overlaps with the frequency of the collision sound of surgical instruments. Conclusion: To develop a sys- 


\section{tem to prevent intraoperative fracture, countermeasures, such as noise canceling, are ne- cessary to prevent false detection of hammering sounds.}

\section{INTRODUCTION}

Total hip arthroplasty (THA) is a general surgery in orthopedics. The number of surgeries performed has markedly increased over the last 30 years. The number of cases of intraoperative fracture started to increase when press fit stems became popular and cementless fixation replaced cement fixation as the main procedure [1-4]. It is presumed that intraoperative fracture is not clinically reported actively because of its negative impact. Including the number of cases not reported, its incidence may therefore be higher.

In the Harvard Medical Practice Study performed in the US State of New York, 100,000 of 180,000 deaths from medical malpractice per year were preventable accidents [5]. The ratio of highly preventable accidents may also be high in THA. When intraoperative fracture in THA was prevented, the duration of hospitalization was shortened to one-sixth of that of fracture cases, which may improve the patient's QOL and largely reduce medical expenses.

Aiming at the prevention of intraoperative fracture, we assessed the force and sounds of hammering a stem into the femur, and searched for an evaluation method that enables the prediction of fracture $[6,7]$. We reported that fixation of the femur and stem cannot be acquired unless the 1st peak frequency of hammering sound (peak frequency) stabilizes, and it may be possible to prevent excess impacting and fracture by judging that fixation has been acquired and refraining from hammering when the peak frequency becomes constant [8-10]. At the second and third peak frequencies, it was not possible to detect problems related to stem fixation. As the 1st peak frequency serves as an important evaluation index, it is necessary to investigate the band of the peak frequency of the hammering sound when fixation is acquired.

The objective of this study was to identify the peak frequency of the hammering sound of impacting the stem into the femur using a hammer in THA. As there may be individual differences in bone quality among patients, we investigated whether the peak frequency changes depending on bone quality. These investigations are the first step in the development of an intraoperative fracture-preventing system in response to on-site needs for accuracy and simplicity. An established system may provide safety and security to both surgeons and patients.

\section{MATERIALS AND METHODS}

This study did not involve humans and did not require IRB approval. To acquire quantitative results, a biomechanical test material (Fourth Generation Cylinder 3403-03-2, Sawbones, WA, USA) was used (Figure 1(A), Figure 1(B)). This is simulated femur prepared by filling a $3-\mathrm{mm}$ thick cortical bone cylinder simulating the physical properties of human bone with 30-pcf cancellous bone [11, 12]. To investigate whether the peak frequency changes due to bone quality, 10 and 30-pcf biomechanical test materials (Fourth Block, Solid Foam, Sawbones, WA, USA) were used based on the bone quality of THA-indicated patients and healthy individuals, respectively (Figure $1(\mathrm{C})$ ). The medullary cavity of the simulated femur was prepared with a rasp and reamer (VerSys, Zimmer Biomet, IN, USA) used in clinical practice.

The simulated femur was fixed to the fixation part of a load test machine for a hip prosthesis with a screw (Figure 2). Hammering sounds generated by impacting the stem using a hammer through an inserter (VerSys, Zimmer Biomet, IN, USA) were analyzed using a fast Fourier transform analyzer (DS-3000, ONOSOKKI, Japan) (Figure 3). The frequency range was set at $10 \mathrm{kHz}$, the resolution was set at $12.5 \mathrm{~Hz}$, the sampling frequency was set at $20.6 \mathrm{kHz}$, and averaging was applied as noise processing.

The distance from the sound source to the directional microphone (Mic FP-5500, SONY, Japan) was set at $1.5 \mathrm{~m}$ based on an operating room. The hammering sound collected using the microphone was converted to a spectrum by a fast Fourier transform analyzer and the frequency at which the intensity reached the maximum was regarded as the peak frequency. The impactor directly impacted by the hammer is 

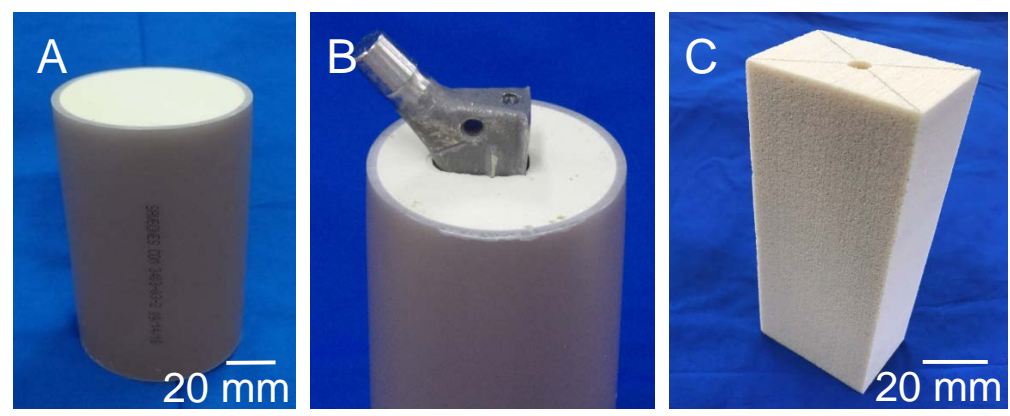

Figure 1. Biomechanical test materials. (A) Cylindrical femur with cortical bone; (B) Cylindrical femur with an implanted stem; (C) Block-type trabecular bone.
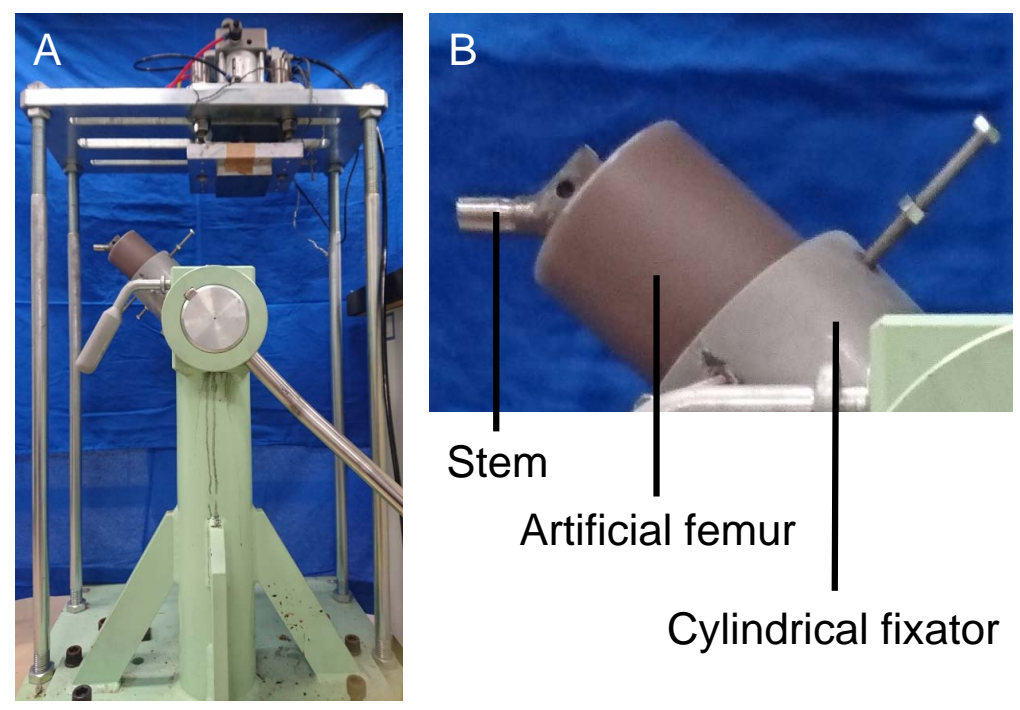

Cylindrical fixator

Figure 2. Environment of hammering sound collection. (A) Loading machine; (B) Enlarged fixation part.

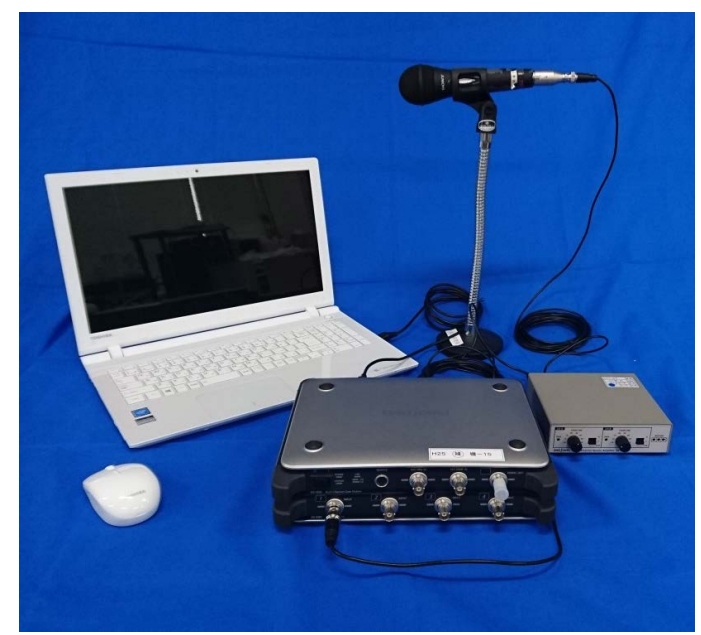

Figure 3. Fast Fourier transform analyzer and direction microphone. 
strongly influenced by its characteristics such as the length and quality of the material. Thus, the peak frequency of the sound generated by impacting the impactor alone with the hammer was measured. These series of procedures were performed for each hammering session and the peak frequency was determined.

The environmental sound level in the operating room was measured using a digital sound level meter (KZ-GM1351, HITACHI, Japan). As many members of orthopedic THA teams are male, sounds were collected from 6 males to represent the operator's voice. Sounds generated by randomly colliding the hammer, impactor, rasp, reamer, and box chisel as surgical instruments mainly used in THA were measured. The hammering sound was compared to investigate whether operator's voice and collision sound of surgical instruments can be distinguished.

\section{RESULTS}

The impactor directly impacted by the hammer is strongly influenced by its characteristics such as the length and quality of the material. Thus, the impactor alone was hammered with the hammer and the peak frequency was $3.41 \pm 0.05 \mathrm{kHz}$ (Figure $4(\mathrm{~A})$ ). The hammering sound is derived from air vibrations caused by the aggregation of femur, stem, impactor, and hammer. The peak frequency of the sound generated by this aggregate was $2.43 \pm 1.45 \mathrm{kHz}$ (Figure 4(B)). When the only impactor was hit and when the aggregate was hit, the spectrum was one of those determined in multiple trials of hammering in both conditions and the frequency was that judged as the acquisition of fixation.

When the same spectrum continued, it was judged as the acquisition of fixation (Figure 5). The peak frequency was identical in these spectra. In the spectrum before acquisition of fixation (from the 1st to 10th), it was not the same.

The peak frequency of the hammering sound of the 10-pcf simulated femur assuming THA-indicated patients was $2.98 \pm 0.73 \mathrm{kHz}$, whereas that of the 30 -pcf simulated femur assuming healthy patients was $2.15 \pm 0.32 \mathrm{kHz}$, that of human voice assuming the surgeon's voice was $0.47 \pm 0.23 \mathrm{kHz}$, and that of the

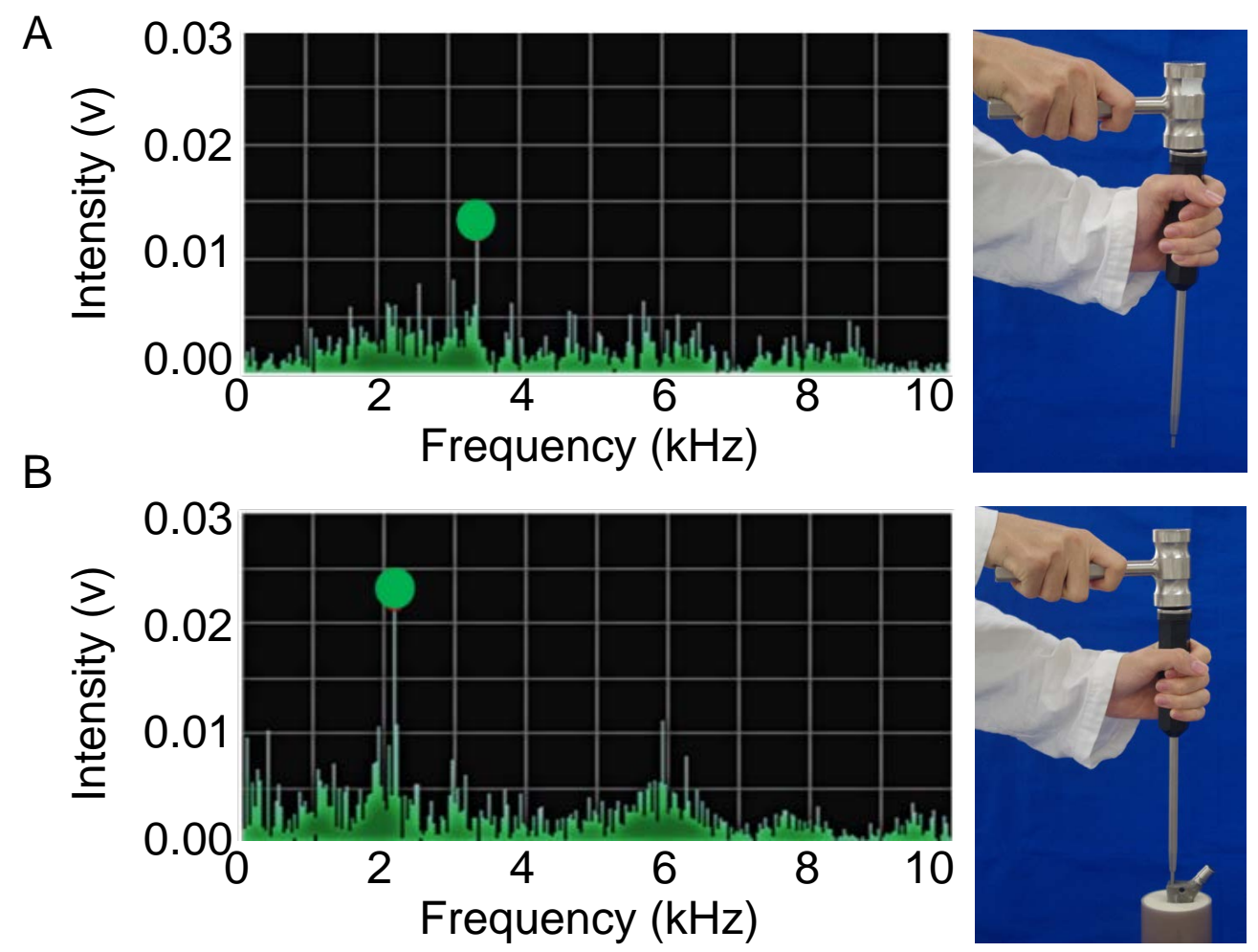

Figure 4. Spectra. Peak frequency is a green dot. (A) Spectrum of the impactor; (B) Spectrum of the impactor, stem, and cylindrical femur. 


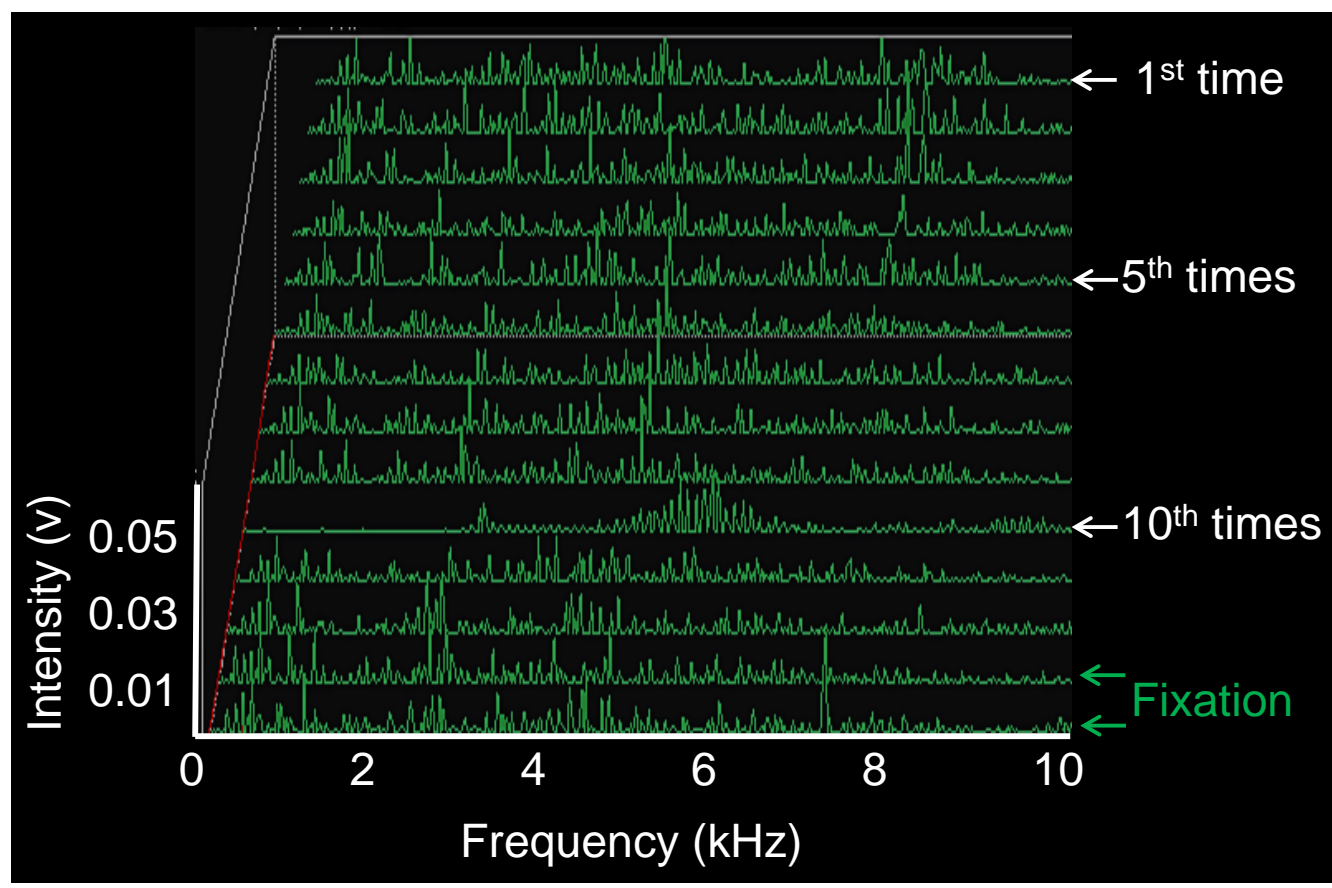

Figure 5. Spectra of the impactor, stem, and cylindrical femur when a stem was implanted into a cylindrical femur. The white arrow indicates the number of hammerings. The green arrow shows a similar spectrum.

collision sound of the surgical instruments was $2.71 \pm 0.52 \mathrm{kHz}$. There was a significant difference in the peak frequency due to the difference in bone quality. The maximum level of environmental sound measurement in the operating room was $24.3 \mathrm{~dB}$ in the voice of the surgeon. The frequency decreased as the bone quality increased, and the environmental sound in the operating room and band overlapped (Figure 6).

\section{DISCUSSION}

The natural frequency of an impactor was previously reported to be $4.5 \mathrm{kHz}$ [13]. The gripping part of the impactor used by Furuichi et al. was made of metal, whereas that used in our study was made of resin, thus the frequency cannot be simply compared [13]. The standard deviation of the peak frequency of the impactor was lower than that of the aggregate of the femur, stem, impactor, and hammer, which may have been due to the presence of fewer influencing elements in the impactor than in the aggregate.

The peak frequency is reported to be below $3 \mathrm{kHz}$ [14]. The sound source depends on not only the collision sound between the hammer and impactor, but also the surrounding medium [15]. We noted changes in the peak frequency due to the state of sample setting. When a sample was set on an experimental desk using a vise, noise was picked up; therefore, the setting was changed to fixation with a screw of the load test machine. Hammering sound collection was performed in a quiet environment with the air conditioner turned off and windows closed, but complete removal of noise was not possible.

The reason for the decrease in the frequency with an increase in the bone quality was hypothesized to be the inverse proportion of the natural frequency of an object to its length, i.e., change in the relative length of the stem and simulated femur united by hammering. The $10 \mathrm{pcf}$ femur is softer than the $30 \mathrm{pcf}$ femur and the stem sinks into the femur, shortening its length. The higher peak frequency than that of the 30 pcf femur was likely caused by this.

The peak frequency in patients indicated for THA can be distinguished from the environmental sound generated by the operator's voice, but partial overlapping with the peak frequency of collision 


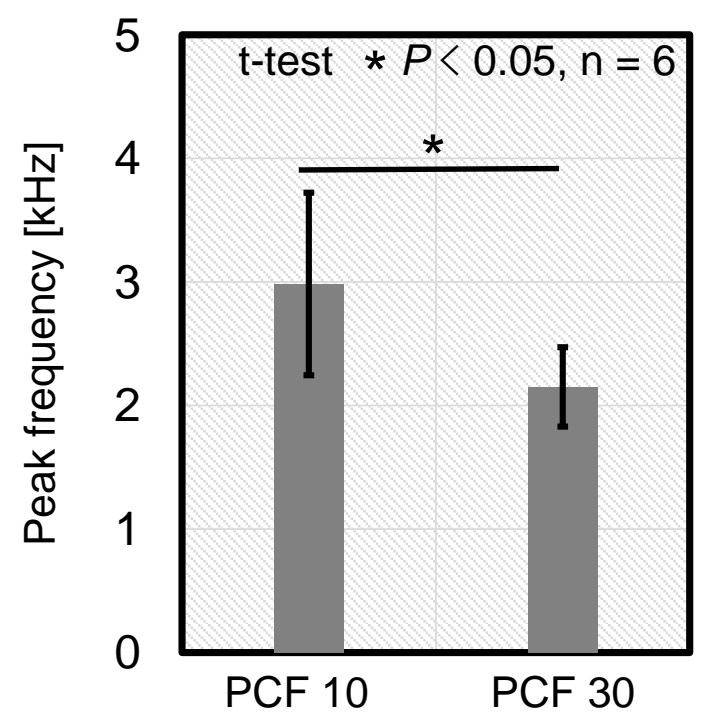

\section{Figure 6. Peak frequency of hammering sound of the impactor, stem, and several trabecular bones (10 pcf, $30 \mathrm{pcf}$ ).}

sounds of surgical instruments was confirmed, for which it is necessary to take countermeasures to prevent false detection. A method of attaching an accelerometer to the grip of the hammer to trigger hammering in order to differentiate the sound from noise was considered, but bringing an accelerometer into the surgical field is not realistic $[16,17]$. The incorporation of active noise canceling producing antiphase waves against metal collision sounds is a realistically possible countermeasure.

There are two limitations in this study. First, the simulated femur did not reflect biological tissue regarding the moisture, length, and absence of adherence from the surrounding tissue. Use of the femur of the affected leg for testing is desirable, but quantitative data cannot be collected; therefore, simulated femur was used. Second, the peak frequency may vary depending on different factors such as the quality of the material, and shape of the stem and impactor. Differences due to these factors can be corrected by incorporating artificial intelligence into the system and utilizing big data.

\section{CONCLUSION}

The peak frequency of hammering sound overlapped with machinery collision sounds in the femur of a similar quality to that in patients indicated for THA. To prevent false detection through the development of an intraoperative fracture-preventing system, countermeasures, such as noise canceling, are necessary.

\section{CONFLICTS OF INTEREST}

The authors declare no conflicts of interest regarding the publication of this paper.

\section{REFERENCES}

1. Berry, D.J. (1999) Epidemiology: Hip and Knee. Orthopedic Clinics of North America, 30, 183-189. https://doi.org/10.1016/S0030-5898(05)70073-0

2. Foster, A.P., Thompson, N.W., Wong, J. and Charlwood, A.P. (2005) Periprosthetic Femoral Fractures-A Comparison between Cemented and Uncemented Hemiarthroplasties. Injury, 36, 424-429. https://doi.org/10.1016/j.injury.2004.07.023

3. Moroni, A., Faldini, C., Piras, F. and Giannini, S. (2000) Risk Factors for Intraoperative Femoral Fractures dur- 
ing Total Hip Replacement. Annales Chirurgiae et Gynaecologiae, 89, 113-118.

4. Schwartz, J.T., Mayer, J.G. and Engh, C.A. (1989) Femoral Fracture during Non-Cemented Total Hip Arthroplasty. The Journal of Bone \& Joint Surgery, 71, 1135-1142. https://doi.org/10.2106/00004623-198971080-00003

5. Paul, C.W., Howard, H.H., Joseph, P.N., William, G.J., Troyen, A.B. and Lucian, L.L. (1993) A Measure of Malpractice: Medical Injury, Malpractice Litigation, and Patient Compensation. Harvard University Press, Cambridge, 4, 99-121.

6. Sakai, R., Takahashi, A., Takahira, N., Uchiyama, K., Yamamoto, T., Uchida, K., Fukusima, K., Moriya, M., Takaso, M., Itoman, M. and Mabuchi, K. (2011) Hammering Force during Cementless Total Hip Arthroplasty and Risk of Microfracture. HIP International, 21, 330-335. https://doi.org/10.5301/hip.2011.8408

7. Sakai, R., Kikuchi, A., Morita, T., Takahira, N., Uchiyama, K., Yamamoto, T., Moriya, M., Uchida, K., Fukusima, K., Tanaka, K., Takaso, M., Itoman, M. and Mabuchi, K. (2011) Hammering Sound Frequency Analysis and Prevention of Intraoperative Periprosthetic Fractures during Total Hip Arthroplasty. HIP International, 21, 718-723. https://doi.org/10.5301/HIP.2011.8823

8. Sakai, R., Yamamoto, T., Uchiyama, K., Takahira, N., Kakeshita, M., Otsu, Y., Yoshida, K. and Ujihira, M. (2020) Prediction of Intraoperative Fracture by Hammering Sound Frequency Analysis and Stress Estimation during Total Hip Arthroplasty. Journal of Biomedical Science and Engineering, 13, 113-119. https://doi.org/10.4236/jbise.2020.136011

9. Sakai, R., Uchiyama, K., Takahira, N., Kakeshita, M., Otsu, Y., Yoshida, K. and Ujihira, M. (2020) Usefulness of Hammering Sound Frequency Analysis as an Evaluation Method for the Prevention of Trouble during Hip Replacement. Journal of Biomedical Science and Engineering, 13, 74-80. https://doi.org/10.4236/jbise.2020.135007

10. Sakai, R., Uchiyama, K., Kensuke, F., Takahira, N., Yoshida, K. and Ujihira, M. (2021) Hammering Sound Frequency Analysis to Fix an Acetabular Cup during Total Hip Arthroplasty: Clinical Trials and Biomechanical Studies. Journal of Biomedical Science and Engineering, 14, 14-20. https://doi.org/10.4236/jbise.2021.141003

11. Jasty, M., Henshaw, R., O'Conner, D. and Harris, D. (1993) High Assembly Strains and Femoral Fracture Produced During Insertion of Uncemented Femoral Components: A Cadaver Study. Journal of Arthroplasty, 8, 479-487. https://doi.org/10.1016/S0883-5403(06)80213-5

12. Herzwurm, P.J., Walsh, J., Pettine, K. and Ebert, F. (1992) Prophylactic Cerclage: A Method of Preventing Femur Fracture in Uncemented Total Hip Arthroplasty. Orthopedics, 15, 143-146.

https://doi.org/10.3928/0147-7447-19920201-06

13. Furuichi, I., Oyama, J. and Endo, M.M. (2013) Analysis of Hammering Sound by Sound Wave Analysis When Inserting Cementless Femoral Stem-8th Reports. Analysis of Metal Impactor Use Group. Japanese Journal of Replacement Arthroplasty, 43, 669-670.

14. Furuichi, I., Oyama, J. and Endo, M.M. (2012) Analysis of Hammering Sound by Sound Wave Analysis When Inserting Cementless Femoral Stem-6th Reports. Study of 3 Cases of Intraoperative Fracture. Japanese Journal of Replacement Arthroplasty, 42, 149-150.

15. Furuichi, I., Oyama, J., Endo, M.M. (2012) Analysis of Hammering Sound by Sound Wave Analysis When Inserting Cementless Femoral Stem-7th Reports. ECHELON. Japanese Journal of Replacement Arthroplasty, 42, 151-152.

16. Rowlands, A., Duck, F.A. and Cunningham, J.L. (2008) Bone Vibration Measurement Using Ultrasound: Application to Detection of Hip Prosthesis Loosening. Medical Engineering \& Physics, 30, 278-284.

https://doi.org/10.1016/j.medengphy.2007.04.017

17. Pastrav, L.C., Jaecques, S.V.N., Jonkers, I., der Perre, G.V. and Mulier, M. (2009) In Vivo Evaluation of a Vibration Analysis Technique for the Per-Operative Monitoring of the Fixation of Hip Prostheses. Journal of Orthopaedic Surgery and Research, 4, Article No. 10. https://doi.org/10.1186/1749-799X-4-10 